\title{
Risk factors for osteoradionecrosis of the jaw in patients with head and neck squamous cell carcinoma
}

\author{
Hikaru Kubota', Daisuke Miyawaki ${ }^{1}$, Naritoshi Mukumoto' ${ }^{1}$ Takeaki Ishihara' ${ }^{1}$, Megumi Matsumura², \\ Takumi Hasegawa ${ }^{2}$, Masaya Akashi ${ }^{2}$, Naomi Kiyota ${ }^{3}$, Hirotaka Shinomiya ${ }^{4}$, Masanori Teshima ${ }^{4}$, Ken-ichi Nibu ${ }^{4}$ \\ and Ryohei Sasaki ${ }^{*}$ (i)
}

\begin{abstract}
Background: To evaluate factors associated with osteoradionecrosis of the jaw (ORNJ) in patients with head and neck squamous cell carcinoma (HNSCC), focusing on jaw-related dose-volume histogram (DVH) parameters.

Methods: We retrospectively reviewed the medical records of 616 patients with HNSCC treated with curative-intent or postoperative radiation therapy (RT) during 2008-2018. Patient-related (age, sex, history of smoking or alcohol use, diabetes mellitus, performance status, pre-RT dental evaluation, pre- or post-RT tooth extraction), tumor-related (primary tumor site, T-stage, nodal status), and treatment-related (pre-RT surgery, pre-RT mandible surgery, induction or concurrent chemotherapy, RT technique) variables and DVH parameters (relative volumes of the jaw exposed to doses of 10 Gy-70 Gy [V10-70]) were investigated and compared between patients with and without ORNJ. The Mann-Whitney U test was used to compare RT dose parameters. Univariate and multivariate Cox regression analyses were used to assess factors associated with ORNJ development. Kaplan-Meier analyses were performed for cumulative ORNJ incidence estimation.

Results: Forty-six patients (7.5\%) developed ORNJ. The median follow-up duration was 40 (range 3-145) months. The median time to ORNJ development was 27 (range 2-127) months. DVH analysis revealed that $\mathrm{V} 30-\mathrm{V} 70$ values were significantly higher in patients with than in those without ORNJ. In univariate analyses, primary tumor site, preRT mandible surgery, post-RT tooth extraction, and V60 > 14\% were identified as important factors. In multivariate analyses, V60 $>14 \%(p=0.0065)$ and primary tumor site $(p=0.0059)$ remained significant. The 3-year cumulative ORNJ incidence rates were $2.5 \%$ and $8.6 \%$ in patients with V60 $\leq 14 \%$ and $>14 \%$, respectively $(p<0.0001)$, and $9.3 \%$ and $1.4 \%$ in patients with oropharyngeal or oral cancer and other cancers, respectively $(p<0.0001)$.
\end{abstract}

Conclusions: $V 60>14 \%$ and oropharyngeal or oral cancer were found to be independent risk factors for ORNJ. These findings might be useful to minimize ORNJ incidence in HNSCC treated with curative RT.

Keywords: Osteoradionecrosis of the jaw, Head and neck squamous cell carcinoma, Dose-volume histogram

*Correspondence: rsasaki@med.kobe-u.ac.jp

${ }^{1}$ Division of Radiation Oncology, Kobe University Hospital, Kobe, Hyogo, Japan

Full list of author information is available at the end of the article

\section{Background}

Osteoradionecrosis of the jaw (ORNJ) is among the most serious late complications observed in patients with head and neck squamous cell carcinoma (HNSCC) treated with radiation therapy (RT). While ORNJ was first described in 1922, the definition, mechanism of 
pathogenesis, incidence, risk factors, and clinical staging, as well as treatment protocols associated with the disease require further investigation [1]. Despite the lack of a standard and unified definition, ORNJ is usually defined as an area of exposed irradiated bone that fails to heal over a period of 3-6 months in the absence of local tumor recurrence [2]. Radiological evidence of bone necrosis within the target volume is also important for the diagnosis and classification of ORNJ severity [3]. Several risk factors of ORNJ have been reported, including patient-related [4-7], tumor-related $[4,5,8,9]$, and treatment-related factors $[5,8,10-16]$.

The reported incidence of ORNJ ranges widely according to the examined periods. The incidence of ORNJ has decreased in recent times, from approximately $20 \%$ several decades ago to $4-8 \%$ in the modern era $[8,13,14$, 17-19]. This tendency might be attributed to improvements in RT techniques, such as the intensity-modulated RT (IMRT), currently used. Several reports have indicated the trend in the rate of jaw-related complications among patients receiving IMRT compared with those receiving 3-dimensional conformal RT (3D-CRT) $[11,15,20]$. In contrast, no reductions were observed in long-term ORNJ rates following IMRT in the absence of attempts to reduce the jaw volumes receiving high doses in IMRT plan optimization [21]. Thus, it is necessary to determine the dose-volumetric threshold of the jaw for IMRT plan optimization to reduce the incidence of ORNJ. Nevertheless, few studies have reported the RT dose-volume correlation of the irradiated jaw [14-16], and the dose-volumetric threshold of ORNJ has not been clearly determined.

The purpose of this study was to identify the risk factors of ORNJ in patients with HNSCC in the modern era, including IMRT. Of the treatment-related factors, the dose-volume histogram (DVH) parameters of the jaw were intensively evaluated with the goal of achieving optimal dose constraints of the jaw and guiding the formulation of future planning objectives.

\section{Methods}

\section{Patient selection}

A total of 616 patients who received definitive-intent or postoperative RT to the head and neck between January 2008 and August 2018 at our institution were included in this retrospective analysis. Data were obtained from patients' medical records, and the inclusion criteria were as follows: age over 20 years, histological confirmation of squamous cell carcinoma, follow-up duration of at least 3 months after RT, completion of the planned RT dose, no previous RT of the head and neck region, and presence of sufficient treatment RT plan data for the evaluation of the dose to the jaw. In this study, HNSCC included nasopharyngeal cancer, oropharyngeal cancer, hypopharyngeal cancer, oral cancer, and cervical lymph node metastases from unknown primary tumors. Patients with laryngeal cancer were not included in this study because the radiation fields for T1-2N0 laryngeal cancer are located completely outside the jaw.

\section{Treatment for HNSCC}

Patients were treated with 3D-CRT (from 2008 to 2014) or IMRT (from 2014 to 2018). Computed tomography (CT) simulation was performed in patients immobilized using a thermoplastic mask. IMRT treatment typically included volumetric-modulated arc therapy using the simultaneous integrated boost (SIB-VMAT) technique. In general, 69.96 or 70 Gy (delivered in 2.12 Gy or 2 Gy per fraction) was prescribed for definitive RT, 50-60 Gy (delivered in 2 Gy per fraction) was prescribed for postoperative RT in cases with minor risk features, and $66 \mathrm{~Gy}$ (delivered in 2 Gy per fraction) was prescribed for postoperative RT in patients with high risk features. Dose to the jaw was not considered during RT planning. In general, tri-weekly cisplatin (dose $80-100 \mathrm{mg} / \mathrm{m}^{2}$ ) was used for concurrent chemoradiotherapy as part of both the definitive and postoperative therapies, while a few patients received concurrent cetuximab, as appropriate. Induction regimens administered to a small number of patients, as needed, also included docetaxel and/or fluorouracil. Before treatment, patients generally underwent pre-RT dental evaluation and management, including tooth extractions, as deemed appropriate by the oral and maxillofacial surgeons, based on risk assessment.

\section{Patient follow-up}

After completing the RT, all patients were evaluated using a video laryngoscope every month for the 1st year, every 2 months for the next 2 years, and every 3 months thereafter for a total of at least 5 years by both radiation oncologists and head and neck surgeons. For oral care and dental evaluation, the patients were generally followed up at 3 and 6 months and at 1, 1.5, and 2 years after completing the RT at the Department of Oral and Maxillofacial Surgery of our hospital. If dental and oral symptoms, such as pain, trismus, and infection, were recorded during the follow-up period, they were initially treated conservatively. However, when the symptoms did not resolve and extraction was the only treatment option, dental extraction was considered.

\section{Definition of ORNJ}

ORNJ was defined as an area of clinically exposed necrotic bone that was present in the radiation fields over a period of 3 months and/or required treatment with surgical intervention or hyperbaric oxygen therapy 
(HBO) without evidence of tumor recurrence [2]. There is also a subset of ORNJ that presents with clinically intact mucosa along with radiographic evidence including evidence derived from CT $[3,13,22,23]$. We included both subsets in our cohort. ORNJ was graded according to the Common Terminology Criteria for Adverse Events v5.0 (CTCAE) [24]. The grades of ORNJ, as defined and graded according to the CTCAE v5.0, include grade 1: asymptomatic; clinical or diagnostic observations only; intervention not indicated; grade 2: symptomatic; medical intervention indicated (e.g., topical agents); limitations in the performance of instrumental activities of daily living (ADLs); grade 3: severe symptoms; limitations in the performance of self-care ADLs; elective operative intervention indicated; grade 4: life-threatening consequences; urgent intervention indicated; and grade 5: death. This study evaluated patients with grade $\geq 2$ ORNJ.

\section{Potential risk factors of ORNJ}

The potential risk factors of ORNJ included patientrelated variables (age, sex, history of smoking, history of alcohol use, diabetes mellitus as a comorbidity, performance status, pre-RT dental evaluation, pre-RT tooth extraction, post-RT tooth extraction), tumor-related variables (primary tumor site, T-stage, nodal status), and treatment-related variables (pre-RT surgery, pre-RT mandible surgery, induction chemotherapy, concurrent chemotherapy, RT technique), and DVH parameters of the jaw.

\section{DVH analysis of the jaw}

The jaw was contoured by one investigator for each plan (Fig. 1), and DVHs were created. The relative volumes of the jaw exposed to doses ranging from 10 to 70 Gy in 10-Gy increments (V10-70) were reviewed as the RT dosimetric parameters. Individual DVH analysis with respect to the location of the ORNJ was also conducted. The maximum dose to the jaw (Dmax), mean dose to the jaw (Dmean), and dose to the $2 \mathrm{cc}$ of the jaw (D2cc) were reviewed for individual DVH analysis. The ORNJ location was reviewed from patients' medical records. All DVH analyses were conducted using Velocity (Varian Medical Systems, Palo Alto, CA, USA).

\section{Statistical analysis}

To compare patient- and treatment-related characteristics in the groups with and without ORNJ, Fisher's test was used to compare the distributions of categorical variables, and $t$ tests were used to compare the distributions of continuous variables. The Mann-Whitney $U$ test was used to compare patients in terms of the presence of ORNJ (present vs. absent), RT technique (3DCRT vs. IMRT), and primary tumor site (oropharyngeal cancer/oral cancer vs. others) in DVH parameters (V1070). Cut-off points for DVH parameters were selected based on the Akaike information criterion (AIC) for the

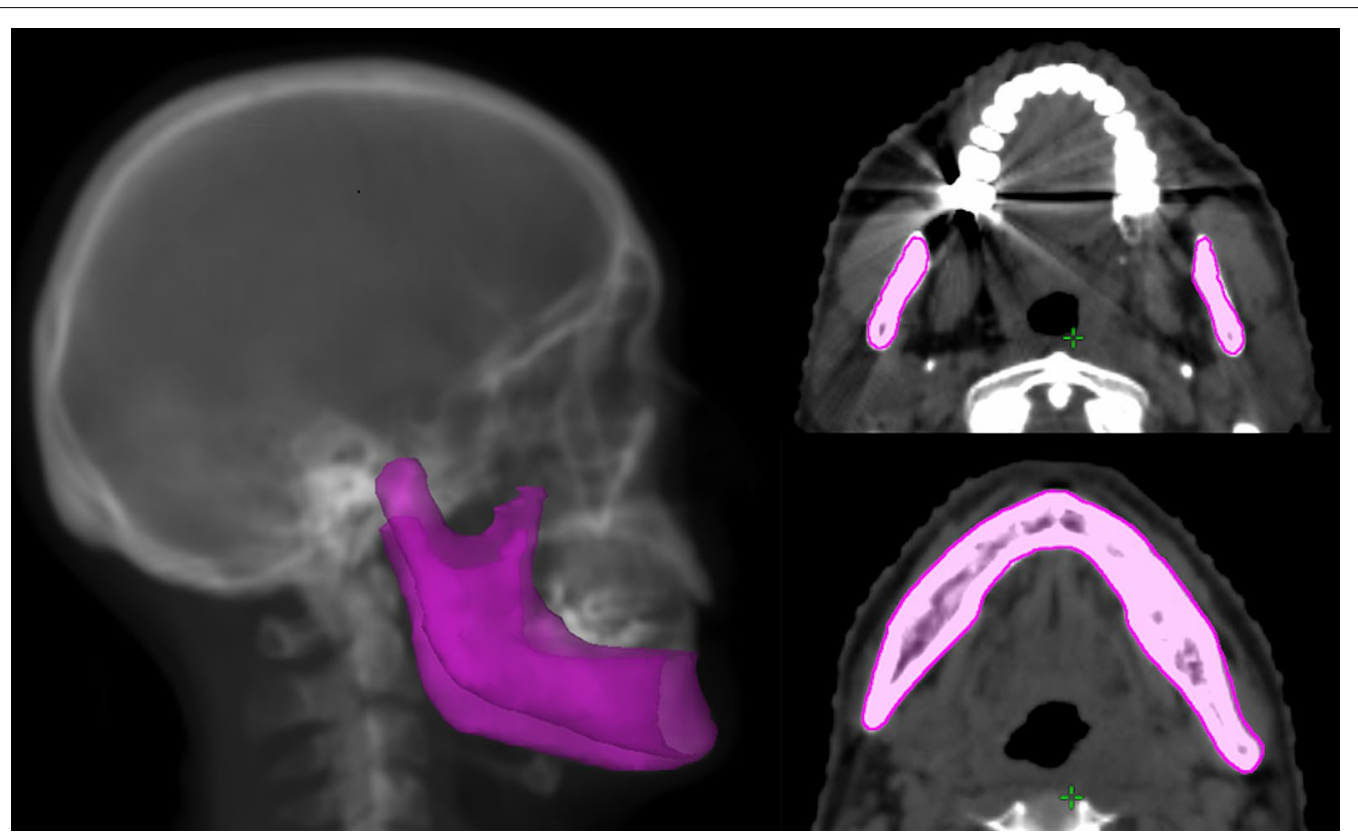

Fig. 1 Representative contouring of the jaw. The whole jaw was contoured including the body, angle or ramus, symphyseal or parasymphyseal, condylar process/head, and coronoid 
development of ORNJ [25]. The AIC is based on in-sample fit for the estimation of the likelihood of a model in predicting future values; the best model is the one with the minimum AIC value among all the other models. Univariate and multivariate Cox proportional hazard models were used to assess patient- and treatment-related factors associated with the development of ORNJ. Factors with statistical significance in the univariate analysis were included in the multivariate analysis. The cumulative incidence was estimated using the Kaplan-Meier method, and the log-rank test was used to compare cumulative incidence curves. The follow-up time was calculated from the last date of RT until the most recent follow-up visit at our institution or the date of death. The time to ORNJ development was calculated from the last date of RT until the date of ORNJ occurrence. All statistical analyses were performed using EZR (Saitama Medical Center, Jichi Medical University, Saitama, Japan), which is a graphical user interface for $\mathrm{R}$ (The $\mathrm{R}$ Foundation for Statistical Computing, Vienna, Austria) [26]. Statistical significance was defined as a $p$ value of 0.05 or less.

\section{Results}

\section{Patient- and treatment-related characteristics}

Among the 616 patients included in the study, the median follow-up time was 40 months (range 3-145 months). ORNJ developed in 46 patients (7.5\%) at 47 sites of the jaw, and the median time to ORNJ development was 27 months (range 2-127 months). The patient- and treatment-related characteristics of patients with and without ORNJ are summarized in Table 1. ORNJ developed more frequently among patients with a primary tumor in the oropharynx or oral cavity $(p<0.0001)$, in those who underwent post-RT tooth extraction $(p<0.0001)$, and in those receiving concurrent chemotherapy $(p=0.0121)$ (Table 1).

In total, $83 \%$ of 47 ORNJ sites were located in the body of the jaw, $43 \%$ were located in the angle or ramus, and $11 \%$ were identified in the symphyseal or parasymphyseal area; ORNJ was not observed in the condylar process/ head or coronoid. In 16 of the ORNJ sites, overlap was observed in the plural subsite of the jaw.

Of the 47 ORNJ sites, bone exposure was observed in 41 (87\%), and cutaneous fistula was seen in 15 (32\%). Both bone exposure and cutaneous fistula were noted in 10 sites (21\%).

CT scans were evaluable for 46 ORNJ sites. On CT, cortical erosion was observed in 38 (83\%) sites, loss of spongiosa trabeculation was noted in $32(70 \%)$ sites, and pathological fracture was observed in $12(26 \%)$ sites.

Of the 46 patients with ORNJ, 22 (47\%) had CTCAE grade 2, 23 (49\%) had grade 3, and two (4\%) had grade 4 ORNJ.
Regarding ORNJ treatment, all patients received conservative management, four $(9 \%)$ received $\mathrm{HBO}$, and 21 (46\%) required surgical management. Of the four patients treated with $\mathrm{HBO}$, two also received additional surgical ORNJ management. At the time of analysis, the disease had been cured or stabilized in 42 (91\%) of the 46 patients.

Of the 46 patients with ORNJ, one patient (2\%) had a history of bisphosphonate treatment.

\section{Review of radiation dose distributions in the ORNJ specific site}

A review of the radiation dose distributions in the 47 ORNJ sites showed that the median Dmax for the ORNJ regions was $68.5 \mathrm{~Gy}$ (range 48.4-77.2 Gy), median D2cc was 62.7 Gy (range 10.8-75.3 Gy), and median Dmean was 62.6 Gy (range 24.4-73.3 Gy).

\section{Dosimetric comparison of the irradiated jaw}

The results of the jaw DVH comparisons are summarized in Table 2. Statistically significant differences were observed between patients with and without ORNJ in the V30 to V70 of the jaw using the nonparametric test. The difference at V60 was the most significant $(12.16 \%$ vs. $35.31 \%, p<0.0001)$. On comparing patients treated with 3D-CRT and IMRT, the V40-70 values were significantly higher with 3D-CRT, whereas the V10-20 values were significantly higher with IMRT. On comparing the primary tumor site, all DVH parameters (V10-70) were significantly higher in oral cancer or oropharyngeal cancer cases than in other primary site cancer cases.

\section{Cut-off DVH parameter values}

To identify optimal cut-off DVH parameter values (V1070) of the jaw for comparing the ORNJ and non-ORNJ groups, the AIC value was calculated for each of these DVH parameters. The AIC value was the lowest at V60 among V10-70 (Fig. 2a). The AIC value was the lowest when the V60 of the jaw was divided by $14 \%$ (Fig. 2b). Therefore, a V60 value of $14 \%$ was decided as the cut-off value. The AIC values for V10-70 are shown in Additional file 1 .

\section{Risk factors associated with the development of ORNJ}

The results of the univariate and multivariate analyses performed for the clinical factors and DVH parameters for the estimation of their effects on the development of ORNJ are summarized in Tables 3 and 4. The univariate analysis demonstrated a significantly increased risk of ORNJ in association with primary tumor site, preRT mandibular surgery, post-RT tooth extraction, and V60 $>14 \%$. Multivariate analyses were performed with primary tumor site (oropharyngeal cancer/oral cancer vs. 
Table 1 Patient, tumor, and treatment characteristics

\begin{tabular}{|c|c|c|c|c|}
\hline Characteristic & $\begin{array}{l}\text { All patients } \\
(n=616)(\%)\end{array}$ & $\begin{array}{l}\text { Non-ORNJ } \\
(n=570)(\%)\end{array}$ & $\begin{array}{l}\text { ORNJ } \\
(n=46)(\%)\end{array}$ & $P$ value \\
\hline \multicolumn{5}{|l|}{ Patient-related factors } \\
\hline Age, years [median (range)] & $65(23-89)$ & $65.5(23-89)$ & $64.5(37-82)$ & 0.2460 \\
\hline \multicolumn{5}{|l|}{ Sex } \\
\hline Male & $513(83)$ & $473(83)$ & $40(87)$ & \\
\hline Female & $103(17)$ & $97(17)$ & $6(13)$ & 0.6810 \\
\hline \multicolumn{5}{|l|}{ History of smoking } \\
\hline No & $464(75)$ & $133(23)$ & $7(15)$ & \\
\hline Yes & $140(23)$ & $426(75)$ & $38(83)$ & 0.2710 \\
\hline Missing & $12(2)$ & $11(2)$ & $1(2)$ & \\
\hline \multicolumn{5}{|l|}{ History of alcohol use } \\
\hline No & $150(24)$ & $139(24)$ & $11(24)$ & \\
\hline Yes & $449(73)$ & $416(73)$ & $33(72)$ & 1.0000 \\
\hline Missing & $17(3)$ & $15(3)$ & $2(4)$ & \\
\hline \multicolumn{5}{|l|}{ Diabetes mellitus } \\
\hline No & $507(82)$ & $470(82)$ & $37(80)$ & \\
\hline Yes & $101(16)$ & $93(16)$ & $8(17)$ & 0.8350 \\
\hline Missing & $8(1)$ & $7(1)$ & $1(2)$ & \\
\hline \multicolumn{5}{|l|}{ Performance status } \\
\hline 0 & $180(29)$ & $161(28)$ & $19(41)$ & \\
\hline 1 & $286(46)$ & $272(48)$ & $14(30)$ & \\
\hline 2 & $83(13)$ & $76(13)$ & $7(15)$ & \\
\hline 3 & $13(2)$ & $13(2)$ & $0(0)$ & 0.0968 \\
\hline Missing & $54(9)$ & $48(8)$ & $6(13)$ & \\
\hline \multicolumn{5}{|l|}{ Pre-RT dental evaluation } \\
\hline No & $128(21)$ & $118(21)$ & $10(22)$ & \\
\hline Yes & $488(79)$ & $452(75)$ & $36(78)$ & 0.8510 \\
\hline \multicolumn{5}{|l|}{ Pre-RT tooth extraction } \\
\hline No & $393(64)$ & $368(65)$ & $25(54)$ & \\
\hline Yes & $223(36)$ & $202(35)$ & $21(46)$ & 0.2020 \\
\hline \multicolumn{5}{|l|}{ Post-RT tooth extraction } \\
\hline No & $567(92)$ & $533(94)$ & $34(74)$ & \\
\hline Yes & $49(8)$ & $37(6)$ & $12(26)$ & $<0.0001$ \\
\hline \multicolumn{5}{|l|}{ Tumor-related factors } \\
\hline \multicolumn{5}{|l|}{ Tumor site } \\
\hline OPC & $169(27)$ & $147(26)$ & $22(48)$ & \\
\hline OC & $161(26)$ & $143(25)$ & $18(39)$ & \\
\hline $\mathrm{HPC}$ & $226(37)$ & $223(39)$ & $3(7)$ & \\
\hline NPC & $42(7)$ & $40(7)$ & $2(4)$ & \\
\hline CUP & $18(3)$ & $17(3)$ & $1(2)$ & 0.0001 \\
\hline \multicolumn{5}{|l|}{ T-stage } \\
\hline T0-2 & $370(60)$ & $338(59)$ & $32(70)$ & \\
\hline T3-4 & $246(40)$ & $232(41)$ & $14(30)$ & 0.2110 \\
\hline \multicolumn{5}{|l|}{ Nodal status } \\
\hline Negative & $131(21)$ & $123(22)$ & $8(17)$ & \\
\hline Positive & $485(79)$ & $447(78)$ & $38(83)$ & 0.5790 \\
\hline \multicolumn{5}{|l|}{ Treatment-related factors } \\
\hline \multicolumn{5}{|l|}{ Pre-RT surgery } \\
\hline No & $378(61)$ & $354(62)$ & $24(52)$ & \\
\hline Yes & $238(39)$ & 216 (38) & $22(48)$ & 0.2090 \\
\hline
\end{tabular}


Table 1 (continued)

\begin{tabular}{|c|c|c|c|c|}
\hline Characteristic & $\begin{array}{l}\text { All patients } \\
(n=616)(\%)\end{array}$ & $\begin{array}{l}\text { Non-ORNJ } \\
(n=570)(\%)\end{array}$ & $\begin{array}{l}\text { ORNJ } \\
(n=46)(\%)\end{array}$ & $P$ value \\
\hline \multicolumn{5}{|l|}{ Pre-RT mandible surgery } \\
\hline No & $565(92)$ & $526(92)$ & $39(85)$ & \\
\hline Yes & $51(8)$ & $44(8)$ & $7(15)$ & 0.0908 \\
\hline \multicolumn{5}{|l|}{ Induction chemotherapy } \\
\hline No & $563(91)$ & $520(91)$ & $43(93)$ & \\
\hline Yes & $53(9)$ & $50(9)$ & $3(7)$ & 0.7870 \\
\hline \multicolumn{5}{|l|}{ Concurrent chemotherapy } \\
\hline No & $172(28)$ & $167(29)$ & $5(11)$ & \\
\hline Yes & $444(72)$ & $403(71)$ & $41(89)$ & 0.0059 \\
\hline \multicolumn{5}{|l|}{ RT technique } \\
\hline 3D-CRT & $448(73)$ & $409(72)$ & $39(85)$ & \\
\hline IMRT & $168(27)$ & $161(28)$ & $7(15)$ & 0.0593 \\
\hline Total radiation dose, Gy [median (range)] & $69.96(50-75)$ & $69.96(50-75)$ & $69.96(50-70)$ & 0.5210 \\
\hline
\end{tabular}

Table 2 Comparison of the dose-volume histograms

\begin{tabular}{|c|c|c|c|c|c|c|c|c|}
\hline Characteristic & & V10 (\%) & V20 (\%) & V30 (\%) & V40 (\%) & V50 (\%) & V60 (\%) & V70 (\%) \\
\hline \multicolumn{9}{|l|}{ ORNJ } \\
\hline No & Median & 92.90 & 86.59 & 76.61 & 65.07 & 41.68 & 12.16 & 0.03 \\
\hline \multirow[t]{2}{*}{ Yes } & Median & 93.99 & 90.29 & 84.16 & 73.48 & 61.37 & 35.31 & 3.22 \\
\hline & $p$ value* & 0.4050 & 0.2340 & 0.0148 & 0.0016 & $<0.0001$ & $<0.0001$ & 0.0041 \\
\hline \multicolumn{9}{|l|}{ RT technique } \\
\hline 3D-CRT & Median & 87.57 & 81.37 & 76.07 & 70.47 & 52.84 & 26.62 & 0.21 \\
\hline \multirow[t]{2}{*}{ IMRT } & Median & 99.42 & 92.92 & 79.39 & 53.95 & 27.30 & 5.33 & 0.00 \\
\hline & $p$ value* & $<0.0001$ & $<0.0001$ & 0.6300 & $<0.0001$ & $<0.0001$ & $<0.0001$ & 0.0001 \\
\hline \multicolumn{9}{|c|}{ Primary tumor site } \\
\hline OPC/OC & Median & 95.04 & 90.24 & 83.21 & 73.87 & 56.26 & 32.03 & 0.00 \\
\hline \multirow[t]{2}{*}{ Others } & Median & 88.99 & 82.00 & 69.43 & 57.59 & 32.86 & 3.98 & 1.19 \\
\hline & $p$ value* & $<0.0001$ & $<0.0001$ & $<0.0001$ & $<0.0001$ & $<0.0001$ & $<0.0001$ & $<0.0001$ \\
\hline
\end{tabular}

* Mann-Whitney U test

V10, V20, V30, V40, V50, V60, and V70, represent relative volumes of the jaw exposed to 10, 20, 30, 40, 50, 60, and 70 Gy, respectively

ORNJ, osteoradionecrosis of the jaw; RT, radiation therapy; 3D-CRT, 3-dimensional conformal radiation therapy; IMRT, intensity-modulated radiation therapy; OPC, oropharyngeal cancer; OC, oral cancer

others), pre-RT mandible surgery, post-RT tooth extraction, and V60 $>14 \%$ (Table 4). V60 > 14\% was found to be significant $(p=0.0065)$ as a dosimetric parameter in the multivariate models. The primary tumor site was also found to be significant $(p=0.0059)$ as a non-dosimetric factor. The cumulative incidence curves for ORNJ, stratified by V60, primary tumor site, pre-RT mandible surgery, and post-RT tooth extraction are shown in Fig. 3. The 3-year cumulative incidence of ORNJ was $2.5 \%$ in patients with $\mathrm{V} 60 \leq 14 \%$ and $8.6 \%$ in those with V $60>14 \%(p<0.0001)$. The 3 -year cumulative incidence of ORNJ was $9.3 \%$ in patients with oropharyngeal cancer or oral cancer and $1.4 \%$ in those with other cancers $(p<0.0001)$. The 3 -year cumulative incidence of ORNJ was $13.8 \%$ in patients with pre-RT mandible surgery and $4.9 \%$ in those without $(p=0.0045)$, and $6.6 \%$ in patients with post-RT tooth extraction and $5.4 \%$ in those without $(p=0.032)$. 

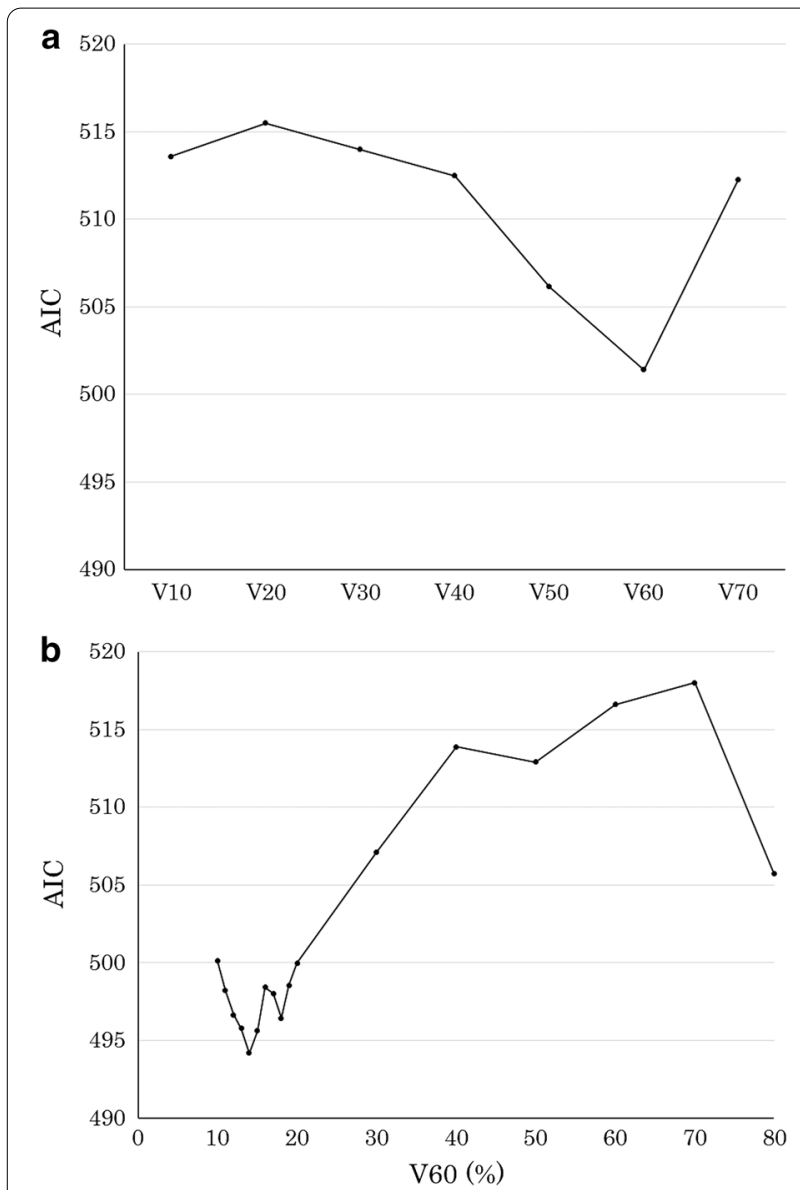

Fig. 2 Akaike information criterion (AIC) value of the volume of the jaw receiving between 10 and $70 \mathrm{~Gy}(\mathbf{a})$, and relationship between AIC and $\mathrm{V} 60$ of the jaw (b). $\mathrm{Vxx}$, relative volumes of the jaw exposed to $x x G y$

\section{Discussion}

In this study, we evaluated the patient-, tumor-, and treatment-related risk factors associated with ORNJ development and found that $\mathrm{V} 60>14 \%$ and oropharyngeal or oral cancer were the most significant risk factors.

ORNJ is among the most serious late morbidities observed in patients with HNSCC with curative RT. With the popularization of IMRT planning, dosimetric assessments of the jaw are now performed to reduce the ORNJ risk. In previous studies, a significantly increased risk of ORNJ was observed in patients receiving high doses (>60-75 Gy) of radiation to the jaw [8, 12-16, 27]. Chen et al. found that a total radiation dose to the primary site of $\geq 75$ Gy was an independent factor associated with ORNJ in 1,692 oral cancer patients [8]. Similarly, Chang et al. demonstrated that radiation doses $\geq 70$ Gy were predictive of ORNJ [27]. Gomez et al. demonstrated that a maximum dose $>70$ Gy and a mean dose $>40$ Gy were associated with an increased rate of subsequent dental
Table 3 Univariate analysis of the development of osteoradionecrosis of the jaw

\begin{tabular}{|c|c|c|c|}
\hline Variable & HR & $95 \% \mathrm{Cl}$ & $P$ value \\
\hline Age & 0.999 & $0.972-1.026$ & 0.9433 \\
\hline Sex & 1.347 & $0.570-3.180$ & 0.4972 \\
\hline History of smoking & 1.489 & $0.664-3.338$ & 0.3336 \\
\hline History of alcohol use & 1.037 & $0.524-2.052$ & 0.9178 \\
\hline Diabetes mellitus & 1.247 & $0.579-2.682$ & 0.5728 \\
\hline Performance status (0-1 vs. 2-4) & 1.608 & $0.707-3.655$ & 0.2573 \\
\hline Pre-RT dental evaluation & 1.280 & $0.623-2.673$ & 0.5017 \\
\hline Pre-RT tooth extraction & 1.469 & $0.819-2.636$ & 0.1971 \\
\hline Post-RT tooth extraction & 2.630 & $1.350-5.125$ & 0.0045 \\
\hline Tumor site (OPC/OC vs. others) & 6.667 & $2.824-15.74$ & $<0.0001$ \\
\hline T-stage (x, 0-2 vs. 3-4) & 0.937 & $0.497-1.768$ & 0.8410 \\
\hline Lymph node positive & 1.408 & $0.657-3.020$ & 0.3794 \\
\hline Pre-RT surgery & 1.713 & $0.960-3.056$ & 0.0687 \\
\hline Pre-RT mandible surgery & 3.053 & $1.356-6.875$ & 0.0070 \\
\hline Induction chemotherapy & 0.748 & $0.232-2.411$ & 0.6267 \\
\hline Concurrent chemotherapy & 2.528 & $0.997-6.411$ & 0.0507 \\
\hline RT technique (3D-CRT vs. IMRT) & 0.886 & $0.380-2.063$ & 0.7789 \\
\hline Total radiation dose & 0.976 & $0.915-1.041$ & 0.4587 \\
\hline V60 ( $\leq 14 \%$ vs. $>14 \%)$ & 6.969 & $2.745-17.700$ & $<0.0001$ \\
\hline
\end{tabular}

$\mathrm{HR}$, hazard ratio; $\mathrm{Cl}$, confidence interval; $\mathrm{RT}$, radiation therapy; $\mathrm{OPC}$, oropharyngeal cancer; OC, oral cancer; 3D-CRT, 3-dimensional conformal radiation therapy; IMRT, intensity-modulated radiation therapy; $\mathrm{V} 60$, relative volumes of the jaw exposed to $60 \mathrm{~Gy}$

Table 4 Multivariate analysis of the development of osteoradionecrosis of the jaw

\begin{tabular}{llll}
\hline Variable & HR & $\mathbf{9 5 \% ~ C l}$ & $\boldsymbol{P}$ value \\
\hline Post-RT tooth extraction & 1.769 & $0.904-3.464$ & 0.0960 \\
Tumor site (OPC/OC vs. others) & 3.577 & $1.443-8.866$ & 0.0059 \\
Pre-RT mandible surgery & 1.714 & $0.753-3.902$ & 0.1988 \\
V60 ( $\leq 14 \%$ vs. > 14\%) & 3.872 & $1.460-10.270$ & 0.0065 \\
\hline
\end{tabular}

$\mathrm{HR}$, hazard ratio; $\mathrm{Cl}$, confidence interval; $\mathrm{RT}$, radiation therapy; $\mathrm{OPC}$,

oropharyngeal cancer; $\mathrm{OC}$, oral cancer; $\mathrm{V} 60$, relative volumes of the jaw exposed to $60 \mathrm{~Gy}$

events and extractions [12], while Aarup-Kristensen et al. reported that the mean dose was significantly associated with ORNJ development [18]. Tsai et al. and Caparrotti et al. suggested that V50 and V60 minimizations were associated with reductions in the rates of ORNJ development in patients with oropharyngeal cancer using a matched case control study $[14,15]$. The MD Anderson Head and Neck Cancer Symptom Working Group showed that most patients with ORNJ have a V $44 \geq 42 \%$ and $\mathrm{V} 58 \geq 25 \%$ in patients with oropharyngeal cancer using RPA analysis in a matched case control study [16]. In the current study, the values of a wide range of 


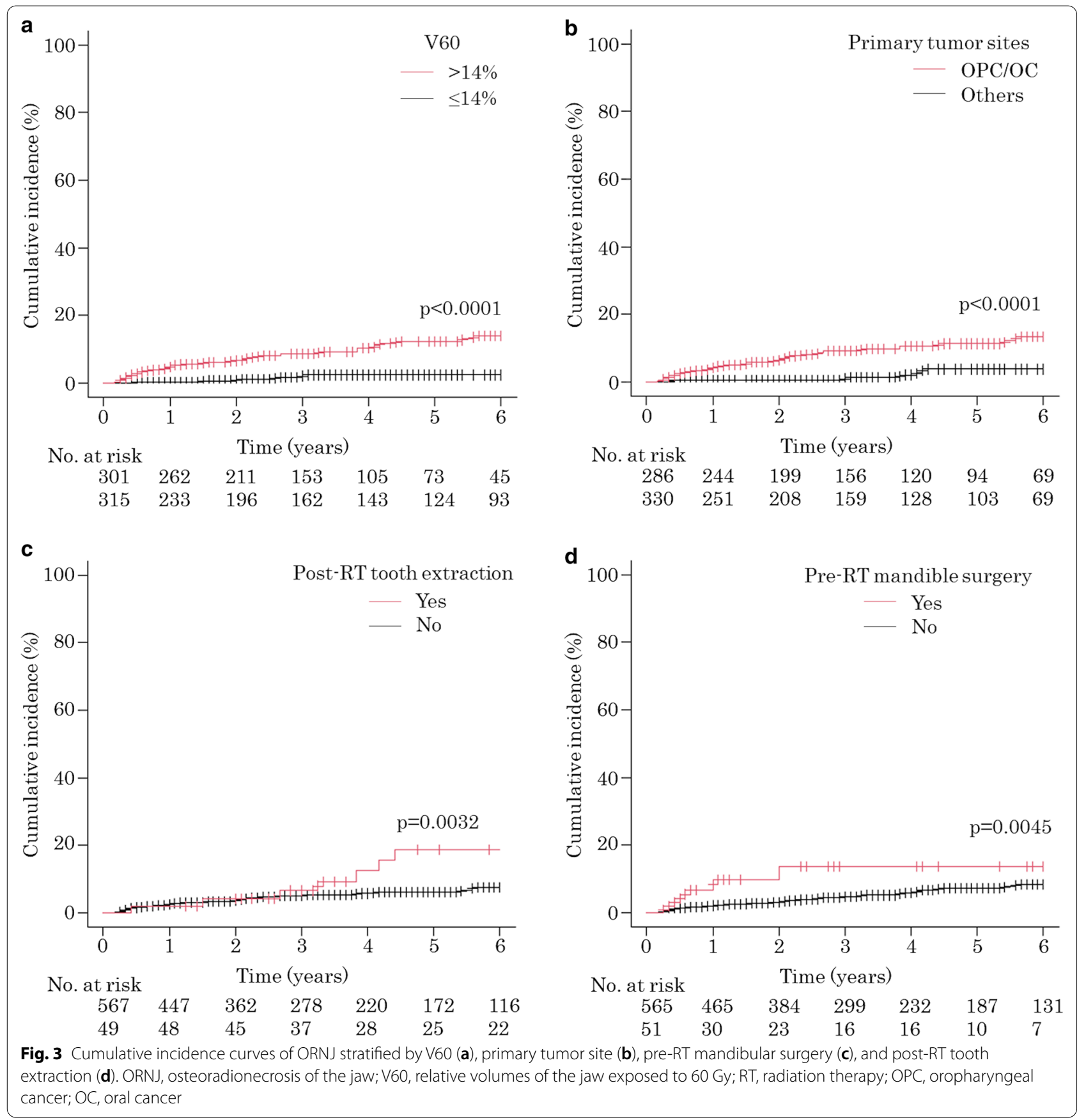

DVH parameters, ranging from V30 to V70, were all significantly higher in the patients with ORNJ than in those without. In our series with a wide spectrum of HNSCC, V $60>14 \%$ was identified as a significant risk factor of ORNJ by both univariate and multivariate analyses. This finding appears to be useful for reducing the risk of ORNJ in daily practice. The cut-off value of V60 appears to be lower than that reported by the MD Anderson Head and Neck Cancer Symptom Working Group (V58 $\geq 25 \%$ ) due to the differences in the included populations. In the evaluation of the specific ORNJ sites, Owosho et al. demonstrated that $96 \%$ of all specific ORNJ sites of the jaw received more than $60 \mathrm{~Gy}$, and the average Dmax was 69.9 Gy (range: 44.3-80.9 Gy) with an average Dmean of 57.4 Gy (range: 28.2-74.6 Gy) [13]. Our results also showed that the median Dmax for the ORNJ regions was 68.5 Gy and the median Dmean was $62.6 \mathrm{~Gy}$, consistent with the findings of Owosho et al. 
The primary tumor site is considered a risk factor for the development of ORNJ, as it represents the anatomical localization of the irradiation volume. Our study showed that primary tumor site was among the most important factors related to ORNJ development. Patients with oral cancer or oropharyngeal cancer have a 4.1-fold risk of ORNJ development compared to patients with other cancers. Similar differences in the relative occurrence frequency of the disease, according to the tumor site, have been reported in the literature [19]. In patients with tumors in the oral cavity or oropharynx, the jaw was at least partially included in the high-dose therapeutic area. In fact, we found that the volume of the irradiated jaw was higher in the oral cavity or oropharynx than in the other tumor sites (Table 2). We recommend including the jaw as an organ at risk during IMRT treatment planning, and the use of IMRT optimization objectives to reduce V60 of the jaw below 14\% without compromising tumor coverage, especially in the treatment of patients with oropharyngeal and oral cancer.

The incidence of ORNJ has decreased in recent times; this can be attributed to improvements in the rates of identification of relevant risk factors and the RT techniques employed. In the present study, $7.5 \%$ of the patients with HNSCC who underwent RT developed ORNJ during the follow-up period, consistent with other recent findings. In general, the incidence of ORNJ varies widely in the literature; this value was as high as $56 \%$ in past decades but has dropped to $4-8 \%$ in recent times $[8,13,14,17-19]$. One of the reasons for this consistent decline in the incidence of ORNJ is the use of IMRT, which potentially allows for better normal tissue sparing rates. In a Surveillance, Epidemiology, and End Results-Medicare study, a downward trend in the rate of jaw complications was observed in those receiving IMRT vs. $3 \mathrm{D}-\mathrm{CRT}(14.0 \%$ vs. $17.3 \%, p=0.064)$ [20]. Similarly, Moon et al. demonstrated significantly lower rates of ORNJ with IMRT use (4.0\% vs. $19 \%, p=0.01)$ in a study including 252 oropharynx cancer and oral cancer patients [11]. However, Maesschalck et al. found no difference in the ORNJ rates between patients treated with IMRT vs. $3 \mathrm{D}-\mathrm{CRT}$ ( $10 \%$ vs. $11 \%$ ); in fact, a higher 3 -year cumulative incidence risk was observed in those receiving IMRT $(8.9 \%$ vs. $4.8 \%, p=0.03)$ [21]. Although the present study did not show that IMRT was superior to 3D-CRT for avoiding ORNJ, it is difficult to conclude that there was no difference between the two methods. Several reasons may be considered. One is that followup duration and dose per fraction were quite different for both methods. Other reasons are that dose to the jaw was not a dose constraint in IMRT planning and that the reduction of high-dose lesions in the jaw was insufficient in patients treated with IMRT. We believe that use of IMRT contributes to the avoidance of ORNJ; we will adopt the protocol to reduce V60 of the jaw to below $14 \%$ in IMRT methods.

Post-RT dental extraction is well-recognized as a patient-related risk factor for ORNJ [28-30]. A systematic review reported that the incidence rate of ORNJ after tooth extraction in irradiated patients was $7 \%$ [31]. Wang et al. suggested that post-RT dental extraction was associated with a gradually increasing risk of ORNJ that peaked at 4-5 years [30]. Poor dental health has also been implicated as a risk factor in the development of ORNJ [13]. Whether pretreatment extraction reduces the rate of ORNJ remains unclear. In our study, pretreatment extraction was performed to avoid the risk of ORNJ. In a previous meta-analysis, patients who received pretreatment extraction had a low ORNJ rate of $4.16 \%$ [32]. A time period of 10-14 days is recommended between pre-RT extraction and irradiation [32]. Muraki et al. reported that dental interventions, including dental evaluation, prophylactic dental extraction, and good dental hygiene maintenance, both before and after RT have strong effects in the prevention of ORNJ development [33]. Efforts should be driven toward the prevention of post-RT dental extraction both before and after RT.

Pre-RT mandible surgery is another treatment-related risk factor associated with ORNJ $[9,19]$. Mandible surgery is often required during the removal of oral cavity tumors adjacent to or infiltrating the mandible. Pre-RT mandible surgery was a risk factor associated with the occurrence of ORNJ in our univariate analysis $(p=0.0055)$; however, its effect was insignificant in the multivariate analysis. Chen et al. reported that patients who underwent segmental mandibulectomy had a higher ORNJ rate compared with those that did not and those who received marginal or hemimandibulectomy [8]. We believe that the extent of surgery and the surgical technique used are also important while considering ORNJ. However, only 51 cases treated with pre-RT mandible surgery were included in this study; further evaluation with a large number of cases is thus necessary.

Along with RT, concurrent chemotherapy offers better local control and overall survival, although it occasionally increases the rates of late toxicity. Whether concurrent chemotherapy increases the risk of ORNJ remains unclear. Reuther et al. observed that ORNJ presented earlier when chemotherapy was used in combination with RT [4]. In contrast, Glanzmann et al. and Wang et al. did not observe increases in the risk of ORNJ with chemotherapy use [30, 34]. In the current study, concurrent chemotherapy had a marginally significant $(p=0.051)$ effect in the univariate analysis. Attention should be paid to the development of ORNJ among patients treated with concurrent CRT. 
In our study, the body (83\%) and angle or ramus (43\%) of the mandible were the most frequent ORNJ sites. Possible reason is that the primary tumor was located close to the body and angle of the mandible in patients with oropharyngeal or oral cancer and close to the high-dose therapeutic area. The blood supply to the mandibular cortex is believed to be an important factor for bone repair. Because the cortex of the body of the mandible is mainly supplied by the periosteal blood flow, insufficient blood supply might be cause of ORNJ occurred at the body of mandible. $[35,36]$.

This study has several limitations. First, due to its retrospective nature, unmeasured confounding variables and potential selection biases could not be accounted for in our analysis. Second, we did not have data on patient oral hygiene conditions that may contribute to ORNJ development. However, we used data on pre- and post-RT dental extractions as surrogates. Additionally, owing to the study design, each tooth could not be individually assessed by the same clinician in the pre- and post-RT period and standardized for tooth extraction indication. Third, the incidence of post-RT xerostomia was not measured in this study, although it is known that RT exposure to the salivary glands can have an impact on the risk of xerostomia and ORNJ [37]. Fourth, of the patients with ORNJ, only one patient $(2 \%)$ had a history of bisphosphonate treatment. It may be preferable to exclude the patients with bisphosphonate because there may be an overlap between medication-related osteonecrosis of the jaw (MRONJ) and ORNJ. Because there were only a small number of cases in our cohort $(n=1 ; 2 \%)$ who had treatment with bisphosphonates, we did not exclude the case from our analyses. Finally, the patients examined were all treated at a single institution. Thus, the extent to which our findings can be generalized to other populations may be limited. However, the current study is the first report of the RT dose-volume correlation of the irradiated jaw in a wide spectrum of HNSCC and worthwhile to be considered in daily practice. An additional prospective study with longer follow-up is needed to confirm our suggestion.

\section{Conclusions}

V $60>14 \%$ and oropharyngeal or oral cancer were determined as the most important risk factors for ORNJ development in the univariate and multivariate analyses. These results can prove useful in decision making for the performance of curative RT for head and neck cancers and, subsequently, V60 $>14 \%$ appears to be useful in daily IMRT planning for reducing the risk of ORNJ development.

\section{Supplementary information}

Supplementary information accompanies this paper at https://doi. org/10.1186/s13014-020-01701-5.

Additional file 1. Cut-off values according to the Akaike's information criterion and dose-volume histogram parameters of the jaw.

\section{Abbreviations}

3D-CRT: 3-Dimensional conformal radiation therapy; ADLs: Activities of daily living; AIC: Akaike's information criterion; CT: Computed tomography; CTCAE: Common Terminology Criteria for Adverse Events v5.0; D2cc: Dose to the 2 cc of the jaw; Dmax: Maximum dose to the jaw; Dmean: Mean dose to the jaw; DVH: Dose-volume histogram; HBO: Hyperbaric oxygen therapy; IMRT: Intensity-modulated radiation therapy; ORNJ: Osteoradionecrosis of the jaw; RT: Radiation therapy; Vxx: Relative volumes of the jaw exposed to xx Gy.

\section{Acknowledgements}

The authors are grateful to the biostatisticians from the Clinical \& Transrational Research Center, Kobe University Hospital for valuable advice concerning the statistical analysis. We would also like to thank Editage (www.editage.jp) for English language editing.

\section{Authors' contributions}

HK and RS conceived and designed the research. HK, DM, NM, MM, and MA collected the data. All authors analyzed and interpreted the data. HK, DM, $\mathrm{TI}$, and RS prepared and wrote the manuscript. MA, TH, NK, HS, MT, and KN collaborated in the discussion and reviewed and revised the manuscript. All authors read and approved the final manuscript.

\section{Funding}

The authors received no specific funding for this work.

\section{Availability of data and materials}

The datasets generated and/or analyzed during the current study are not publicly available because they contain personal information but are available from the corresponding author on reasonable request.

\section{Ethics approval and consent to participate}

Ethical approval was obtained from the Human Research Ethics Committee of our institute (No. 180300). Patients treated at our institution consented in writing for the use of their anonymized data for research purposes.

\section{Consent for publication}

Not applicable.

\section{Competing interests}

The authors declare that they have no competing interests.

\section{Author details}

${ }^{1}$ Division of Radiation Oncology, Kobe University Hospital, Kobe, Hyogo, Japan. ${ }^{2}$ Department of Oral and Maxillofacial Surgery, Kobe University Graduate School of Medicine, Kobe, Japan. ${ }^{3}$ Kobe University Hospital Cancer Center, Kobe, Japan. ${ }^{4}$ Department of Otolaryngology-Head and Neck Surgery, Kobe University Graduate School of Medicine, Kobe, Japan.

Received: 20 August 2020 Accepted: 29 October 2020

Published online: 05 January 2021

\section{References}

1. Chronopoulos A, Zarra T, Ehrenfeld M, Otto S. Osteoradionecrosis of the jaws: definition, epidemiology, staging and clinical and radiological findings. A concise review. Int Dent J. 2018;68:22-30.

2. Lyons A, Ghazali N. Osteoradionecrosis of the jaws: current understanding of its pathophysiology and treatment. Br J Oral Maxillofac Surg. 2008;46:653-60. 
3. He Y, Liu Z, Tian Z, Dai T, Qiu W, Zhang Z. Retrospective analysis of osteoradionecrosis of the mandible: proposing a novel clinical classification and staging system. Int J Oral Maxillofac Surg. 2015;44:1547-57.

4. ReutherT, Schuster T, Mende U, Kübler A. Osteoradionecrosis of the jaws as a side effect of radiotherapy of head and neck tumour patients-a report of a thirty year retrospective review. Int J Oral Maxillofac Surg. 2003:32:289-95.

5. Nabil S, Samman N. Risk factors for osteoradionecrosis after head and neck radiation: a systematic review. Oral Surg Oral Med Oral Pathol Oral Radiol. 2012;113:54-69.

6. Goldwaser BR, Chuang SK, Kaban LB, August M. Risk factor assessment for the development of osteoradionecrosis. J Oral Maxillofac Surg. 2007:65:2311-6

7. Hoff AO, Toth B, Hu M, Hortobagyi GN, Gagel RF. Epidemiology and risk factors for osteonecrosis of the jaw in cancer patients. Ann NY Acad Sci. 2011:1218:47-54.

8. Chen JA, Wang CC, Wong YK, Wang CP, Jiang RS, Lin JC, et al. Osteoradionecrosis of mandible bone in patients with oral cancer-associated factors and treatment outcomes. Head Neck. 2016;38:762-8.

9. Monnier Y, Broome M, Betz M, Bouferrache K, Ozsahin M, Jaques B. Mandibular osteoradionecrosis in squamous cell carcinoma of the oral cavity and oropharynx: incidence and risk factors. Otolaryngol Head Neck Surg. 2011;144:726-32.

10. Sathasivam HP, Davies GR, Boyd NM. Predictive factors for osteoradionecrosis of the jaws: a retrospective study. Head Neck. 2018;40:46-54.

11. Moon DH, Moon SH, Wang K, Weissler MC, Hackman TG, Zanation AM, et al. Incidence of, and risk factors for, mandibular osteoradionecrosis in patients with oral cavity and oropharynx cancers. Oral Oncol. 2017;72:98-103.

12. Gomez DR, Estilo CL, Wolden SL, Zelefsky MJ, Kraus DH, Wong RJ, et al. Correlation of osteoradionecrosis and dental events with dosimetric parameters in intensity-modulated radiation therapy for head-and-neck cancer. Int J Radiat Oncol Biol Phys. 2011;81:e207-13.

13. Owosho AA, Tsai CJ, Lee RS, Freymiller H, Kadempour A, Varthis $\mathrm{S}$, et al. The prevalence and risk factors associated with osteoradionecrosis of the jaw in oral and oropharyngeal cancer patients treated with intensitymodulated radiation therapy (IMRT): the Memorial Sloan Kettering Cancer Center experience. Oral Oncol. 2017:64:44-51.

14. Tsai CJ, Hofstede TM, Sturgis EM, Garden AS, Lindberg ME, Wei Q, et al. Osteoradionecrosis and radiation dose to the mandible in patients with oropharyngeal cancer. Int J Radiat Oncol Biol Phys. 2013;85:415-20.

15. Caparrotti F, Huang SH, Lu L, Bratman SV, Ringash J, Bayley A, et al. Osteoradionecrosis of the mandible in patients with oropharyngeal carcinoma treated with intensity-modulated radiotherapy. Cancer. 2017;123:3691-700

16. MD Anderson Head and Neck Cancer Symptom Working Group. Dosevolume correlates of mandibular osteoradionecrosis in Oropharynx cancer patients receiving intensity-modulated radiotherapy: results from a case-matched comparison. Radiother Oncol. 2017:124:232-9.

17. Studer G, Bredell M, Studer S, Huber G, Glanzmann C. Risk profile for osteoradionecrosis of the mandible in the IMRT era. Strahlenther Onkol. 2016;192:32-9.

18. Aarup-Kristensen S, Hansen CR, Forner L, Brink C, Eriksen JG, Johansen J. Osteoradionecrosis of the mandible after radiotherapy for head and neck cancer: risk factors and dose-volume correlations. Acta Oncol. 2019;58:1373-7.

19. Kuhnt T, Stang A, Wienke A, Vordermark D, Schweyen R, Hey J. Potential risk factors for jaw osteoradionecrosis after radiotherapy for head and neck cancer. Radiat Oncol. 2016;11:101.

20. Beadle BM, Liao KP, Chambers MS, Elting LS, BuchholzTA, Kian Ang K, et al. Evaluating the impact of patient, tumor, and treatment characteristics on the development of jaw complications in patients treated for oral cancers: a SEER-Medicare analysis. Head Neck. 2013;35:1599-605.
21. Maesschalck T, Dulguerov N, Caparrotti F, Scolozzi P, Picardi C, Mach N, et al. Comparison of the incidence of osteoradionecrosis with conventional radiotherapy and intensity-modulated radiotherapy. Head Neck. 2016;38:1695-702.

22. Støre G, Boysen M. Mandibular osteoradionecrosis: clinical behaviour and diagnostic aspects. Clin Otolaryngol Allied Sci. 2000;25:378-84.

23. Owosho AA, Kadempour A, Yom SK, Randazzo J, Jillian Tsai C, Lee NY, et al. Radiographic osteoradionecrosis of the jaw with intact mucosa: proposal of clinical guidelines for early identification of this condition. Oral Oncol. 2015;51:e93-6

24. NIH. National Cancer Institute. DCTD Division of Cancer Treatment \& Diagnosis. CTEP Cancer Therapy Evaluation Program. 2020. https:// ctep.cancer.gov/protocolDevelopment/electronic_applications/ctc. htm\#ctc_50.

25. Akaike $\mathrm{H}$. Information theory and an extension of the maximum likelihood principle. In: Petrov BN, editor. Second international symposium on information theory. Budapest: Akademiai Kiado; 1973. p. 267-81.

26. Kanda Y. Investigation of the freely available easy-to-use software "EZR" for medical statistics. Bone Marrow Transplant. 2013;48:452-8.

27. Chang DT, Sandow PR, Morris CG, Hollander R, Scarborough L, Amdur RJ, et al. Do pre-irradiation dental extractions reduce the risk of osteoradionecrosis of the mandible? Head Neck. 2007;29:528-36.

28. Jereczek-Fossa BA, Orecchia R. Radiotherapy-induced mandibular bone complications. Cancer Treat Rev. 2002;28:65-74.

29. Kuo TJ, Leung CM, Chang HS, Wu CN, Chen WL, Chen GJ, et al. Jaw osteoradionecrosis and dental extraction after head and neck radiotherapy: a nationwide population-based retrospective study in Taiwan. Oral Oncol. 2016;56:71-7.

30. Wang TH, Liu CJ, Chao TF, Chen TJ, Hu YW. Risk factors for and the role of dental extractions in osteoradionecrosis of the jaws: a national-based cohort study. Head Neck. 2017;39:1313-21.

31. Nabil S, Samman N. Incidence and prevention of osteoradionecrosis after dental extraction in irradiated patients: a systematic review. Int J Oral Maxillofac Surg. 2011;40:229-43.

32. Jiang $Y, Z$, hu $X$, Qu S. Incidence of osteoradionecrosis in patients who have undergone dental extraction prior to radiotherapy: a systematic review and meta-analysis. J Oral Maxillofac Surg Med Pathol. 2014:26:269-75.

33. Muraki Y, Akashi M, Ejima Y, Hasegawa T, Miyawaki D, Shinomiya $H$, et al. Dental intervention against osteoradionecrosis of the jaws in irradiated patients with head and neck malignancy: a single-arm prospective study. Oral Maxillofac Surg. 2019;23:297-305.

34. Glanzmann C, Gratz KW. Radionecrosis of the mandibula: a retrospective analysis of the incidence and risk factors. Radiother Oncol. 1995;36:94-100.

35. Saka B, Wree A, Anders L, Gundlach KK. Experimental and comparative study of the blood supply to the mandibular cortex in Göttingen minipigs and in man. J Craniomaxillofac Surg. 2002;30:219-25.

36. Akashi M, Hashikawa K, Wanifuchi S, Kusumoto J, Shigeoka M, Furudoi S, et al. Heterogeneity of necrotic changes between cortical and cancellous bone in mandibular osteoradionecrosis: a histopathological analysis of resection margin after segmental mandibulectomy. Biomed Res Int. 2017:2017:3125842

37. Wang $X, H u C$, Eisbruch A. Organ-sparing radiation therapy for head and neck cancer. Nat Rev Clin Oncol. 2011:8:639-48.

\section{Publisher's Note}

Springer Nature remains neutral with regard to jurisdictional claims in published maps and institutional affiliations. 